\title{
IMPRO4: UNA APLICACIÓN DE TIPO SAAS (SOFTWARE AS A SERVICE) PARA LA EVALUACIÓN DE IMPACTO AMBIENTAL
}

\author{
IMPRO4 - SAAS FOR ENVIRONMENTAL IMPACT ASSESSMENT \\ Teresa Gómez Villarino \\ Ingeniero Agrónomo, Ph.D., Profesor Asociado, Escuela Técnica Superior de \\ Ingenieros Agrónomos, Universidad Politécnica de Madrid. \\ Director Melissa Consultoria e Ingeniero Ambiental SL, Madrid, España. \\ teresagomez@melissa-sa.com \\ Domingo Gómez Orea \\ Ingeniero Agrónomo, Ph.D., Catedrático de Universidad, Escuela Técnica Superior de Ingenieros \\ Agrónomos, Universidad Politécnica de Madrid, Madrid, España. \\ domingo.gomez.orea@gmail.com
}

Fecha de recepción: 28 de marzo de 2012

Fecha de aprobación: 29 de noviembre de 2012

\section{RESUMEN}

Este artículo presenta el programa IMPRO4-EIA, aplicación informática diseñada expresamente para realizar el Estudio de Impacto Ambiental de un proyecto y sus alternativas, en forma sistemática, rápida, eficaz y eficiente, de acuerdo con el contenido que fija la legislación vigente y con el estilo de los más modernos enfoques. Dicho estudio supone el principal soporte técnico de la Evaluación Impacto Ambiental (EIA). El manejo del programa por parte del usuario sigue un proceso conversacional, de tal modo que permite simular distintas hipótesis de trabajo (cambiar criterios, valoraciones, juicios, etc.), obteniendo respuestas rápidas. Dicha posibilidad es de gran interés, dada la incertidumbre inherente a los estudios de impacto ambiental derivada de su carácter predictivo, de su complejidad y de la necesidad de incluir juicios de valor durante todo el proceso de elaboración, debido a que una de las formas más útiles de tratar la incertidumbre, consiste en hacer análisis de sensibilidad, es decir, analizar cómo cambian los resultados cuando se cambian las hipótesis de trabajo, lo cual sólo puede hacerse de manera eficaz cuando se dispone de una herramienta que permita obtener tales resultados con rapidez.

Palabras clave: aplicación informática, evaluación de impacto ambiental, estudio de impacto ambiental, proyecto. 


\begin{abstract}
This paper introduces IMPRO4-EIA, software designed to develop the Environmental Impact Analysis of a project and its alternatives. It works in a systematic, fast, effective and efficient way in compliance with the current rules and latest approaches. This study is the main support of the Environmental Impact Assessment (EIA). The operation of this program is based on a conversational method in order to reproduce several work hypothesis, which enables the user to take into account several criteria, assessments, judgments... obtaining results very quickly. Its complex nature inherent in the course of its process' uncertainty makes this procedure of great interest. And this because one of the most useful ways of dealing with uncertainty is to carry out sensitivity analysis, i.e. to analyze how the results change as working hypotheses change which can only be done effectively with the proper tool to get such results quickly.
\end{abstract}

Keywords: software, environmental impact assessment, environmental impact analysis, project.

\title{
INTRODUCCIÓN
}

La Evaluación de Impacto Ambiental (EIA), es un procedimiento administrativo que apoyándose en un Estudio de Impacto Ambiental y en un proceso de información pública, desemboca en un pronunciamiento de la administración ambiental denominado declaración de impacto ambiental, en el cual se rechaza o se acepta, con o sin condiciones, el proyecto en función de su impacto ambiental. El programa Impro4-EIA se ha diseñado expresamente para realizar Estudios de Impacto Ambiental de proyectos.

El modelo IMPRO es un programa informático que surgió hace más de 20 años para facilitar la aplicación de la metodología diseñada por el profesor Domingo Gómez Orea [1] para realizar estudios de impacto ambiental. Posteriormente, se elaboró una segunda versión, el programa Impro2, que además contenía módulos destinados a generar y evaluar alternativas. Hace unos diez años, se desarrolló la versión Impro3 que desde entonces, se ha venido aplicando profusamente en los ámbitos profesional y académico con excelentes resultados, aplicación que le proporciona una alta solvencia conceptual, técnica e informática.

Los antecedentes metodológicos del modelo IMPRO son los modelos automatizados de planificación (GRID, INGRID, IRANS, modelo de Impacto-Aptitud, MAUSAR), y las metodologías generales de impacto ambiental basadas en matrices (matriz de Leopold, Matriz de Grandes Presas y Sistema Batelle) [2-7].

Pero debido a la rapidez con la cual evoluciona, la informática ha sugerido esta nueva y revolucionaria versión, el Impro4-EIA, que como sus antecesores, puede interpretarse como una aplicación informatizada de la metodología de Gómez Orea para realizar los estudios de impacto

TERESA GÓMEZ VILLARINO, DOMINGO GÓMEZ OREA 
ambiental de cualquier proyecto, garantizando el contenido que se exige a este tipo de estudios, contribuyendo a su calidad y liberando tiempo de tareas repetitivas para destinarlo a los aspectos más sustantivos.

Para concluir esta introducción, conviene advertir la diferencia entre los conceptos de efecto e impacto que se utilizan en el texto; el primero identifica simplemente la alteración de un factor ambiental a causa de una acción; mientras que el impacto ambiental se refiere a la interpretación del efecto en términos de salud y bienestar humano.

\section{PRINCIPALES CARACTERÍSTICAS DE IMPRO4}

Una de las novedades de esta nueva versión, reside en el aprovechamiento de las tecnologías de comunicación existentes, y en concreto Internet: el programa trabaja en entorno web, y reside en un servidor accesible a través del portal http://mww.eisystems.es. Este portal proporciona información sobre el programa, las preguntas más frecuentes en relación con su uso (sugeridas por el uso de sus antecedentes: Improl, 2 y 3 y sus versiones), así como otros servicios que se pueden prestar.

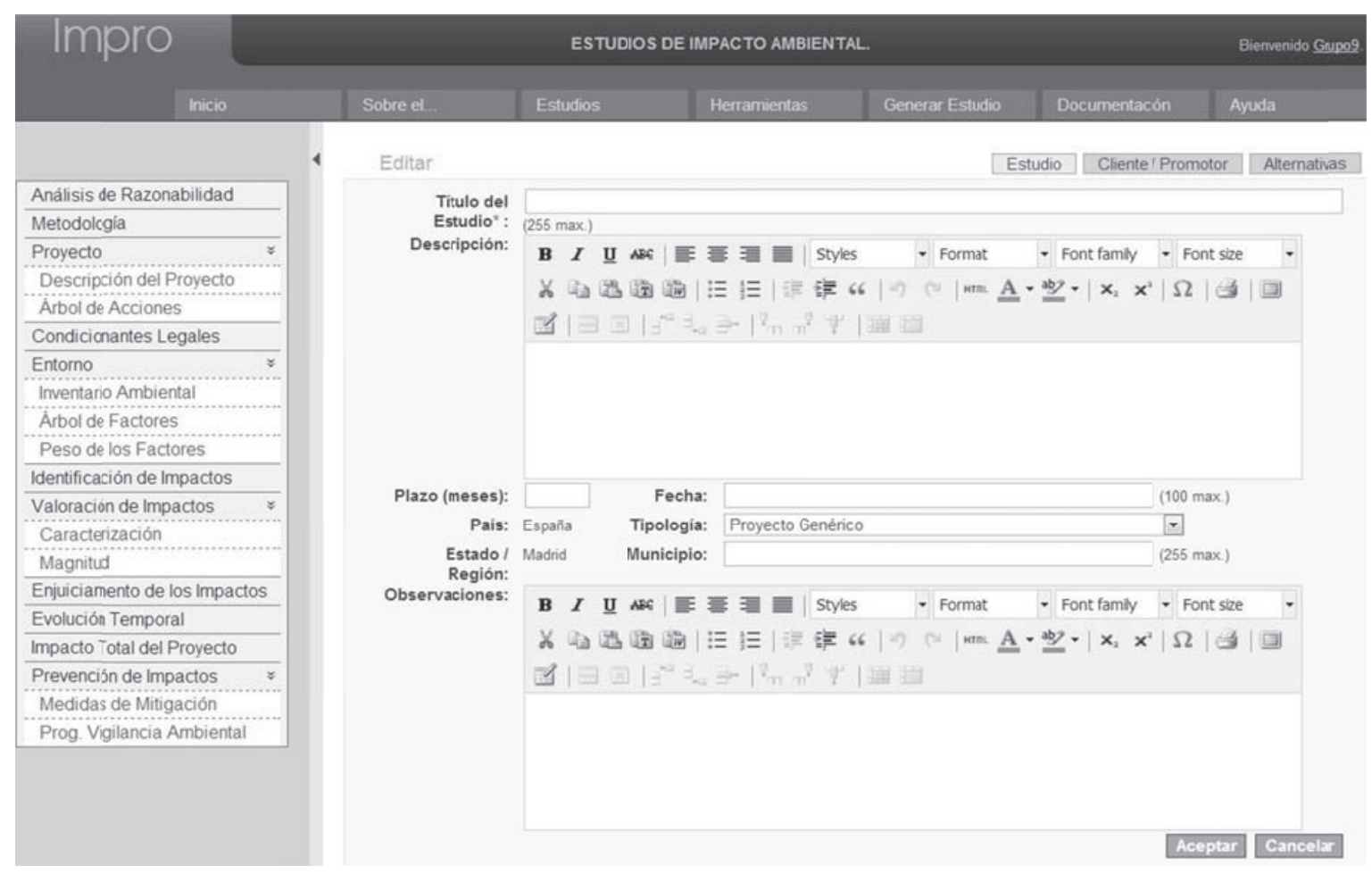

Figura 1. Pantalla principal de la aplicación Impro4 
El contenido legalmente exigido a un estudio de impacto ambiental consta de los siguientes puntos:

1. Descripción del proyecto y sus acciones

2. Examen de alternativas técnicamente viables y justificación de la solución adoptada

3. Inventario ambiental y descripción de las interacciones ecológicas o ambientales claves

4. Identificación y valoración de impactos, tanto en la solución propuesta como en sus alternativas

5. Establecimiento de medidas protectoras y correctoras

6. Programa de vigilancia ambiental

7. Documento de síntesis

IMPRO4-EIA da respuesta a este contenido, organizándolo a partir de una ventana principal (Figura 1). Se dispone de dos menús, uno superior para las opciones genéricas de navegación y principales de cada estudio, y otro menú lateral donde se encuentran agrupadas todas las acciones disponibles para el desarrollo del estudio.

Menú superior: está formado por varias pestañas que dan acceso a las siguientes opciones: Inicio / Sobre el Estudio / Herramientas / Generar Estudio / Documentación / Ayuda.

Menú lateral: está compuesto por los diferentes bloques o componentes del Estudio de Impacto Ambiental: Análisis de razonabilidad o pertinencia del proyecto en su entorno / Metodología / Proyecto / Entorno / Identificación de Impactos / Valoración de los Impactos identificados / Enjuiciamiento de los Impactos / Evolución Temporal /Prevención de Impactos / Impacto Total del Proyecto.

Los bloques anteriores se desagregan en tareas más concretas. La metodología que utiliza Impro4 para realizarlas, sigue una secuencia lineal, pero su aplicación debe hacerse en un proceso de "ir y venir" de unas a otras tareas, es decir, que en todo momento se puede volver atrás para modificar criterios o datos existentes, introducir datos nuevos e incluso, cambiar algunas de las características por defecto del modelo.

\section{SECUENCIA METODOLÓGICA QUE UTILIZA IMPRO4}

A continuación, se describe someramente la secuencia de operaciones para realizar un Estudio de Impacto Ambiental con el programa Impro4, que se organiza genéricamente como muestra la Figura 2:

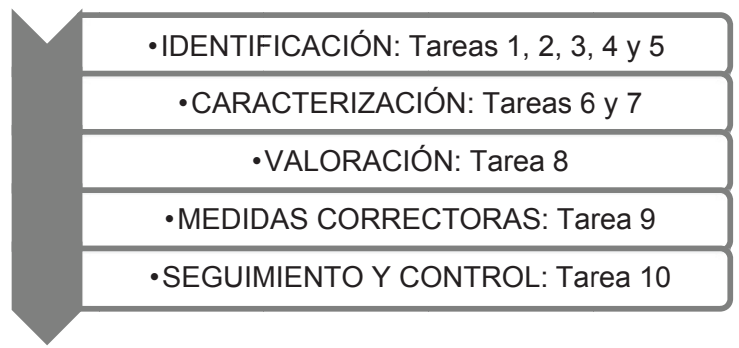

Figura 2. Secuencia de trabajo de la aplicación Impro4

TERESA GÓMEZ VILLARINO, DOMINGO GÓMEZ OREA 
Las siguientes figuras ilustran las tareas más importantes del procedimiento de trabajo con el programa que debe seguir el usuario, y que son las siguientes:

Tarea 1. Elegir el proyecto con el cual se va a trabajar (Figura 3). En este momento, se ofrecen dos nuevas opciones que son: la creación de la ficha de Promotor del Proyecto y, en el caso de que fuese necesario, la posibilidad de incluir hasta cinco alternativas de proyectos por cada Estudio de Impacto.

Tarea 2. Con el fin de simplificar sustancialmente el trabajo, se permite la importación de Alternativas y/o Estudios ya creados por el usuario o de aquellos genéricos que existen en la base de datos.

Tarea 3. Definir un árbol de acciones del proyecto susceptibles de producir impactos. Esta tarea supone desagregar el proyecto en fases (de formulación del proyecto, de construcción, de explotación, de abandono o desmantelamiento, en su caso), elementos (partes bien diferenciadas del proyecto: accesos, edificios, instalaciones, lugares de extracción o deposición, etc.), y acciones causa de impacto (extracción de influentes, alteración del espacio, emisiones, etc.).

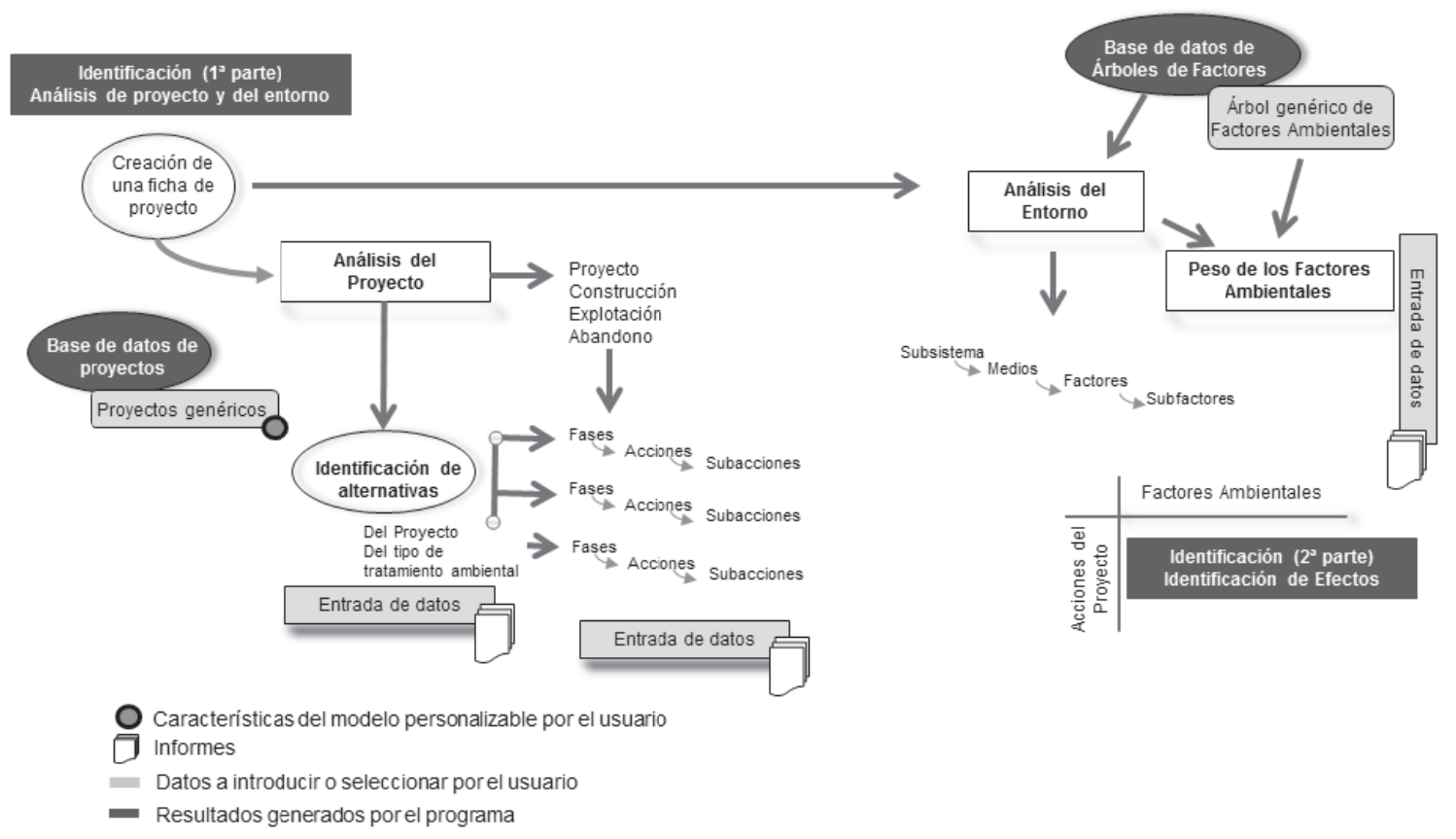

Figura 3. Ilustración de la metodología de Impro4

Tarea 4. Definir un árbol de factores del entorno susceptibles de recibir impactos. Por factores del entorno, se entienden los elementos, cualidades y procesos que pueden verse afectados por las acciones del proyecto; su organización en forma de árbol, facilitará el entendimiento del entorno como un sistema. Esta tarea es análoga a la anterior y para formalizarla, el usuario puede 
generar su propio árbol de factores o adaptar uno cualquiera importado de los disponibles en el programa. Además, esta tarea permite asignar pesos a los factores ambientales, entendiendo por tales, su contribución relativa a la calidad ambiental del entorno (Figura 3).

Tarea 5. Identificar los efectos ambientales. Para identificar un efecto en la aplicación Impro4, se debe relacionar una acción del proyecto con un factor ambiental en el cruce correspondiente (Figura 4).

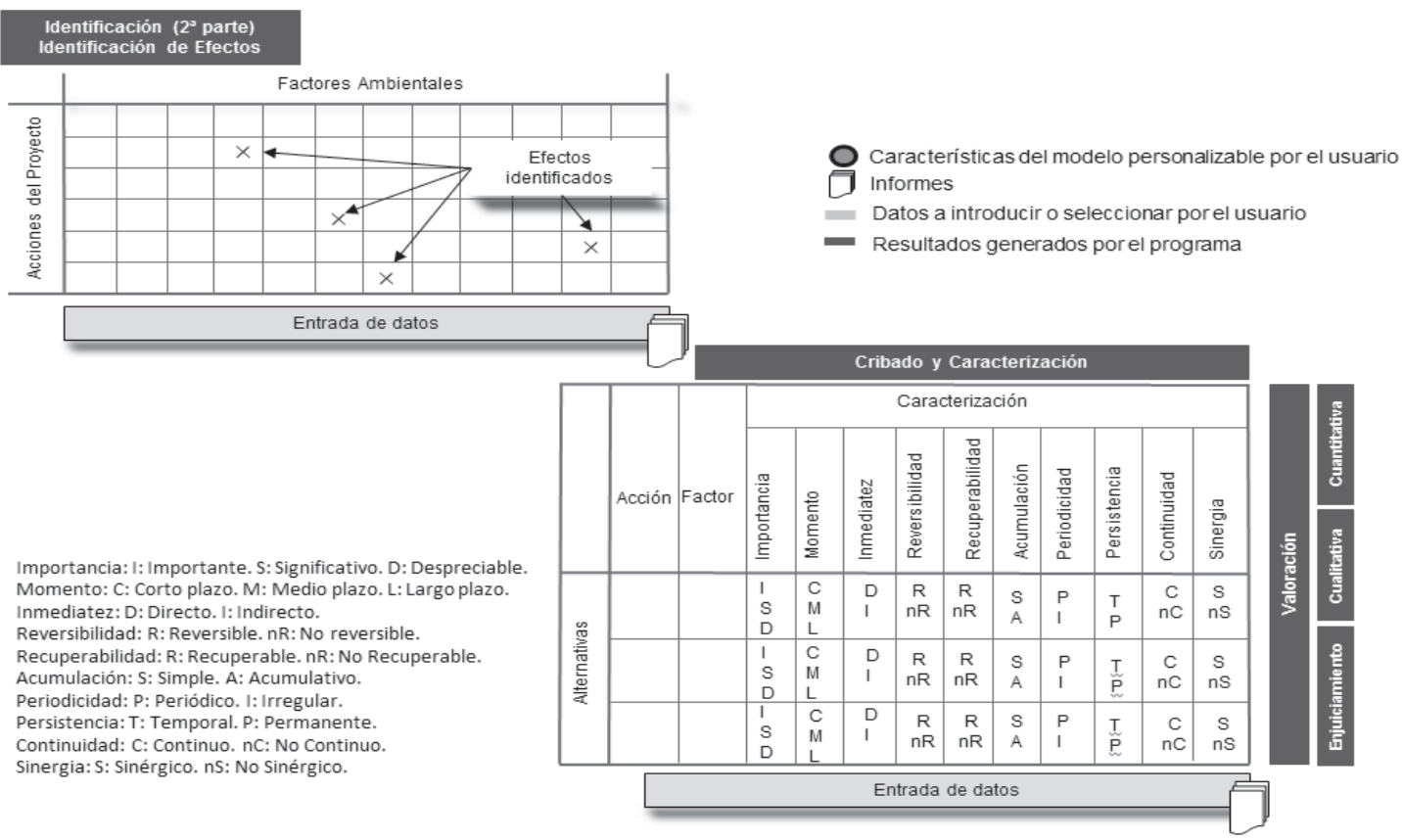

Figura 4. Identificación y caracterización de efectos

Tarea 6. En seguida, se caracterizan los efectos identificados según los atributos que requiere la legislación vigente' (Figura 4), Hernández Fernandez, S. [8]. En esta tarea, también se define el tipo de efecto que se produce y su condición de significativo ${ }^{2}$ o no. Además de una descripción en función de sus principales atributos, la caracterización del impacto constituye una primera parte de su valoración.

Tarea 7. Valorar los Impactos. Supone predecir e interpretar, en términos de calidad ambiental, las alteraciones que la acción causa al factor ambiental. El programa Impro4 permite realizar dos tipos

1 En España, el Real Decreto 1131 del 30 de septiembre de 1988, por el cual se aprueba el Reglamento para la ejecución del Real Decreto legislativo 1302 del 28 de junio de 1986, sobre evaluación de impacto ambiental, establece que para la caracterización del efecto se utilizarán los siguientes atributos: positivo/negativo; temporal/permanente; simple/acumulativo/sinérgico; directo/indirecto; reversible/irreversible; recuperable/irrecuperable; periódico/irregular; continuo/discontinuo.

2 Por efecto significativo se entiende aquel que el Real Decreto 1131 de 1988 define como efecto notable: Aquél que se manifiesta como una modificación del medio ambiente, de los recursos naturales o de sus procesos fundamentales de funcionamiento, que produzca o pueda producir en el futuro, repercusiones apreciables en los mismos. 
de valoración: cuantitativa (Figura 5), y cualitativa (Figura 6). Los impactos se totalizan para todo el proyecto por suma ponderada, haciendo intervenir los pesos asignados a los factores.

\section{Valoración Cuantitativa}

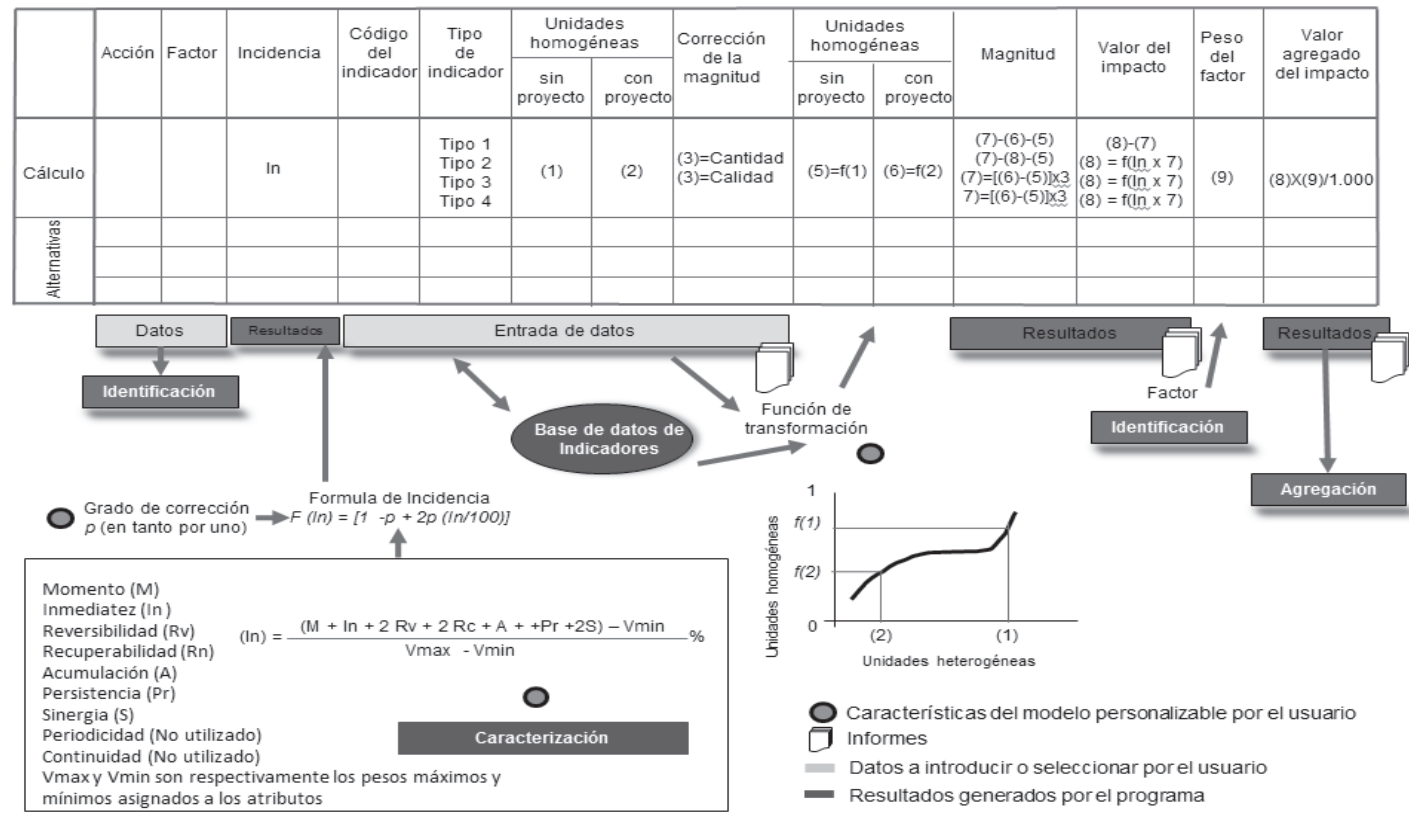

Figura 5. Valoración cuantitativa

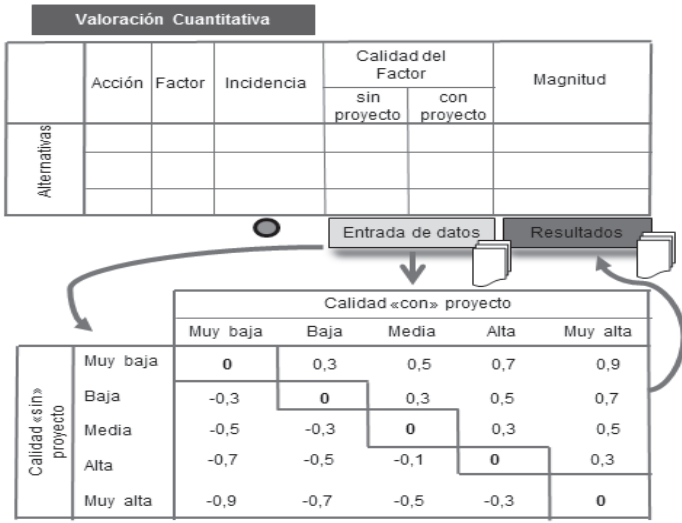

\begin{tabular}{|c|c|c|}
\hline \multicolumn{2}{|c|}{ Categorias } & \multirow{2}{*}{$\begin{array}{l}\text { Características del modelo personalizable por el usuario } \\
\text { Informes }\end{array}$} \\
\hline 0,9 Pos. Muy Importante & $-0,1$ Pos. Neg. Muy Mod. & \\
\hline 0,7 Pos. Importante & $-0,3$ Neg. Moderado & Datos a introducir o seleccionar por el usuario \\
\hline 0,5 Pos. Medio & $-0,5$ Neg. Medio & - Resultados generados por el programa \\
\hline 0,3 Pos. Moderado & $-0,7$ Neg. Importante & \\
\hline 0,1 Pos. Muy Moderado & ${ }^{-0,9}$ Pos. Muy Imp. & \\
\hline
\end{tabular}

Figura 6. Valoración cualitativa 
Tarea 8. Teniendo en cuenta los datos presentados (introducidos y calculados), se emite un juicio acerca de cada impacto, en términos de las categorías definidas por la legislación vigente (Figura 7): positivo, compatible, moderado, severo o crítico.

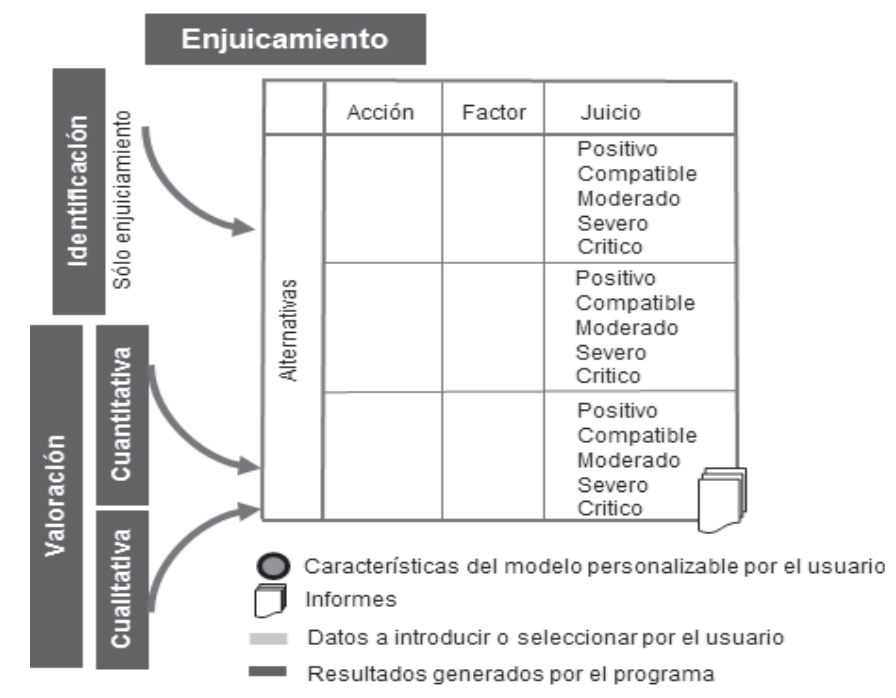

Figura 7. Juicio sobre el impacto: Positivo, compatible, moderado, severo o crítico

Tarea 9. Definir las medidas protectoras, correctoras y compensatorias que se proponen, y describirlas por sus principales características.

Tarea 10. Programa de Vigilancia Ambiental: elaboración del documento de seguimiento y control que contiene el conjunto de criterios técnicos e indicadores que permitirán al promotor y a la administración, realizar un seguimiento sistemático tanto del cumplimiento de lo estipulado en la Declaración de Impacto Ambiental, como de aquellas otras alteraciones de difícil previsión que pudieran aparecer.

A continuación, se puede definir nuevas alternativas del proyecto y copiar en ellas, los efectos de la alternativa base. Según las características de esa alternativa, se eliminan o introducen nuevos impactos y se repiten las valoraciones que sean diferentes. Se realiza lo mismo para el juicio y las medidas correctoras. De nuevo, se repiten las fases anteriores para estas alternativas.

Por último, la herramienta Visualización y generación del Estudio de Impacto Ambiental permite visualizar y verificar los datos introducidos y obtener el "esqueleto" del Estudio propiamente dicho, el cual deberá completarse después, con aspectos de carácter formal como por ejemplo: Introducción, Legislación aplicable, Documento de síntesis, anexos fotográficos, anexos de planos, etc. El usuario puede incluir en este último, aquellos datos de los visualizados, que estime oportuno para ser exportados en formato “.doc" y procesados con cualquier editor de texto. 


\section{DESCRIPCIÓN DETALLADA DEL PROGRAMA}

A continuación, se describe detalladamente todo el sistema de trabajo con Impro4.

\subsection{Alternativas del proyecto}

Impro4 permite trabajar con varias alternativas de un mismo proyecto, lo cual facilita:

1. Incorporar las modificaciones o variantes que se considere oportuno: de localización, tamaño, materiales, tecnología, calendario, etc.

2. Aplicar el programa a la situación "sin" o alternativa "0": no hacer el proyecto, y compararla con la situación "con" proyecto.

3. Definir múltiples alternativas de corrección de los impactos y analizar su eficacia (reducción del impacto), y eficiencia (reducción del impacto por unidad monetaria invertida en el tratamiento de prevención o corrección).

También se puede comparar varios niveles de corrección de impactos desde opciones someras hasta corrección intensiva, analizando la eficacia y eficiencia de la inversión que comporta. Esto facilita la integración ambiental del proyecto en fases previas a la EIA, permitiendo una mayor colaboración entre los técnicos encargados de la redacción del proyecto y los responsables de su evaluación ambiental.

\subsection{Análisis de razonabilidad}

La razonabilidad juzga en qué medida, el proyecto está justificado, es decir, es razonable o pertinente, en el entorno en donde se ubica. El análisis de razonabilidad se realiza mediante las siguientes reglas, cada una de las cuales exige responder una serie de preguntas, (Tabla 1) Gómez Orea [9]:

Tabla 1. Preguntas para realizar el análisis de razonabilidad

Regla 1. Relación con los problemas, necesidades, aspiraciones y expectativas de la población de su entorno: ¿Están identificados los problemas y necesidades de la población en el entorno del proyecto?

¿Atiende el proyecto a los problemas y necesidades de la población de su entorno?

Regla 2. Relación con las aspiraciones de la población

¿Están identificadas las aspiraciones de la población en el entorno del proyecto?

¿Atiende el proyecto las aspiraciones de la población?

Regla 3. Aprovechamiento de recursos naturales endógenos

¿Están identificados los recursos endógenos naturales del entorno del proyecto?

¿Utiliza el proyecto los recursos endógenos naturales del entorno?

Regla 4. Aprovechamiento de recursos construidos endógenos

¿Están identificados los recursos endógenos construidos del entorno del proyecto?

¿Utiliza el proyecto los recursos endógenos naturales del entorno?

Regla 5. Aprovechamiento de recursos humanos endógenos

¿Están identificados los recursos endógenos humanos del entorno del proyecto?

¿Utiliza el proyecto los recursos endógenos humanos del entorno? 


\section{Regla 6. Aprovechamiento de las oportunidades de localización}

¿Están identificadas las oportunidades de localización del entorno del proyecto?

¿Utiliza el proyecto las oportunidades de localización de su entorno?

\section{Regla 7. Integración territorial}

¿Se conoce el papel del entorno del proyecto en el contexto territorial en donde se inscribe?

¿Contribuye el proyecto a la integración territorial de su ámbito?

\section{Regla 8. Integración sectorial}

¿Se conoce el contexto de los sectores socioeconómicos en donde se inscribe el proyecto?

¿Se integra el proyecto en su contexto sectorial?

Al finalizar, se obtendrá como resultado, si el proyecto es razonable y en qué grado lo es, en función del número de reglas que cumple; además, se describen sintéticamente las razones de tal juicio.

\subsection{Definición del proyecto y sus acciones}

El Menú Proyecto (Figura 8), gestiona las tareas relacionadas con la Descripción del proyecto y la creación del Árbol de Acciones. Cuando se realiza esta elección, aparecen las diferentes casillas con los elementos básicos del proyecto (Alternativas, Objetivos, Descripción, etc.), tal y como se muestra en la figura 8.

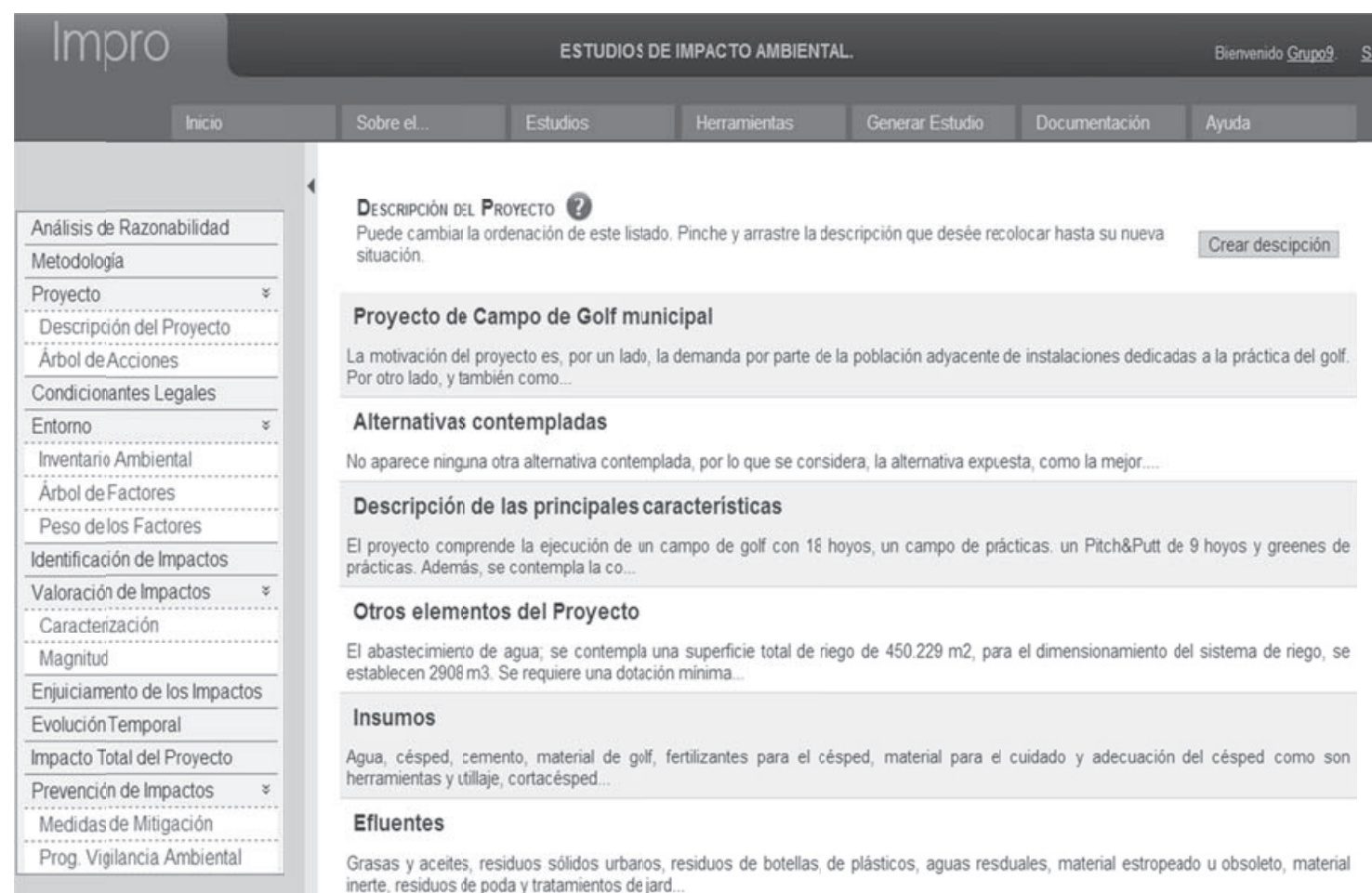

Figura 8. Ventana de Descripción del Proyecto

TERESA GÓMEZ VILLARINO, DOMINGO GÓMEZ OREA 
Las acciones del proyecto susceptibles de producir impactos en el entorno se organizan en forma de árbol con tres niveles. La correcta definición de árboles de acciones del proyecto facilita mucho la fase de identificación de impactos. El árbol de acciones queda incluido en la entrada por columnas de la matriz de identificación de impacto (Figura 9).
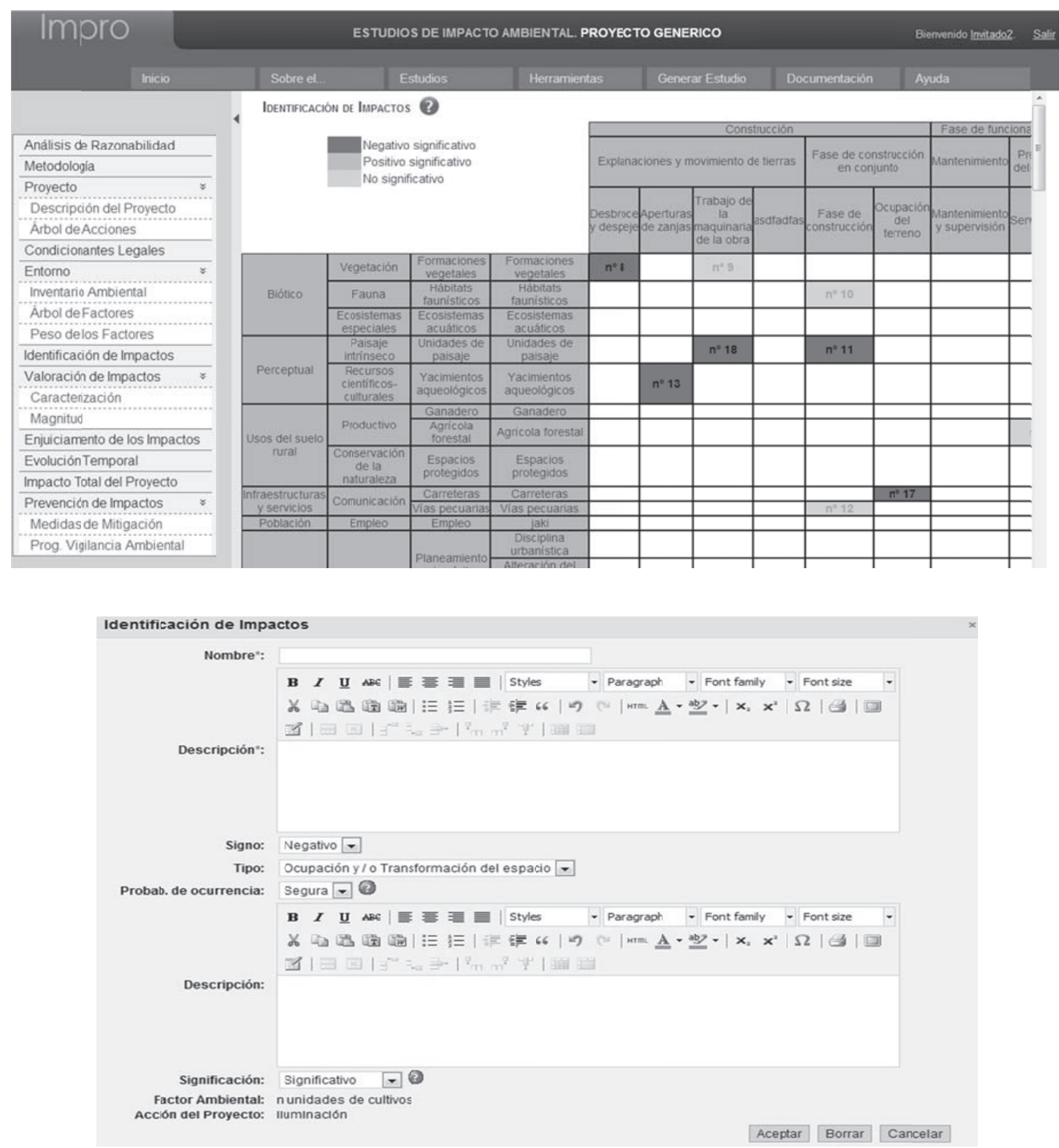

Figura 9. Matriz de identificación de impacto (columnas: árbol de acciones del proyecto y filas: árbol de factores del entorno), y ventana emergente con el formulario de identificación de impacto 


\subsection{Definición del entorno y sus factores.}

El Menú de Entorno gestiona las tareas relacionadas con la descripción del Inventario Ambiental, la composición del Árbol de Factores y su Peso o importancia en el entorno.

Los factores ambientales susceptibles de recibir impactos, se organizan en forma de árbol, con un máximo de cuatro niveles. A diferencia del árbol de acciones que puede ser diferente para cada alternativa, el árbol de factores ambientales es común para todas las alternativas del proyecto, y la ventana es similar, salvo por los cuatro niveles de profundidad: subsistema, medio, factor y subfactor.

Peso de los factores ambientales. Los pesos de cada factor representan su contribución relativa al total de la calidad ambiental. El método para asignar pesos que utiliza la aplicación Impro4, consiste en repartir 1.000 puntos en cada nivel. El usuario irá repartiendo puntos por cada casilla en blanco que considere relevante; el programa calcula de forma automática, los porcentajes para cada factor.

\subsection{Identificación de impactos}

Esta tarea consiste en predecir las interacciones proyecto-entorno. Para tal fin, Impro4 opera, ayudando a formalizar matrices de impacto clásicas, donde las columnas y filas están formadas por acciones del proyecto y factores ambientales, respectivamente.

Para identificar una relación, basta con pinchar un cruce de fila con columna. Se abrirá entonces, una ventana emergente con los datos necesarios para identificar el impacto, como se muestra en la Figura 9.

Como se puede ver en la Figura 9, la identificación se realiza en esta misma ventana, en donde además, se describe el impacto identificado en términos de los atributos que marca la legislación. Así mismo, se hace un cribado del impacto, asignándolo a uno de los siguientes grupos: significativo positivo, significativo negativo o despreciable.

\subsection{Valoración de impactos}

En la aplicación Impro4, la valoración de impactos puede ser cuantitativa (mediante indicadores de impacto y funciones de transformación), o cualitativa (utilizando rangos para establecer la calidad del factor "sin" y "con" proyecto).

La valoración cuantitativa opera mediante dos índices: el de incidencia que se deduce de la caracterización de cada impacto, a partir de los valores atribuidos a cada atributo que la conforman, y el de magnitud, que se determina cualitativa o cuantitativamente, a partir de la cantidad y calidad del factor alterado. 
Bajo la opción Valoración de Impactos, existen dos submenúes que permiten caracterizar y valorar los impactos identificados.

Caracterización de impactos: cálculo de la incidencia del impacto. El programa calcula automáticamente el valor de la incidencia a partir de la caracterización de cada efecto (Figura 10), y muestra su valor estandarizado entre 0 y 1 en la parte superior izquierda de cada casilla identificada (Figura 13). El manual del programa y sus ayudas, explican los valores numéricos que se dan a cada atributo, la ecuación utilizada para obtener la Incidencia y la manera de estandarizarla.

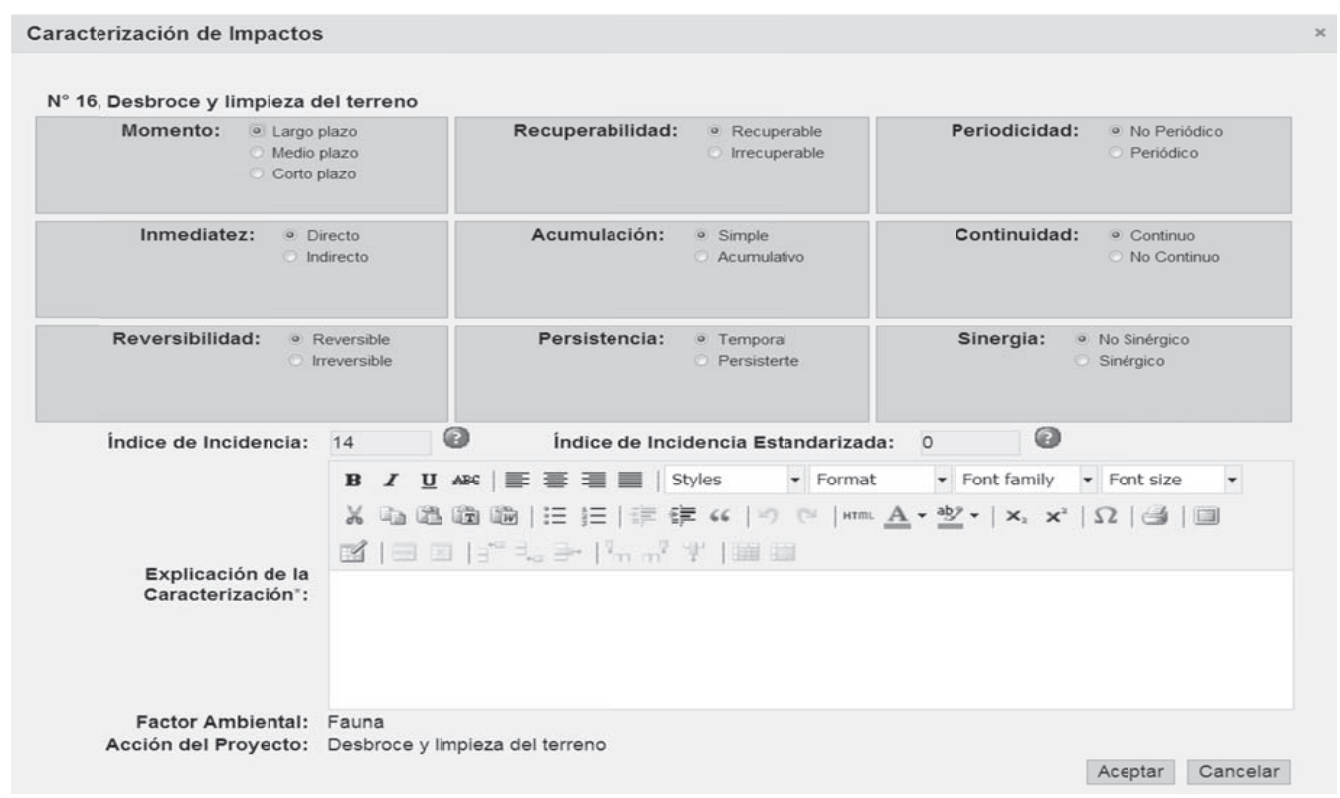

Figura 10. Obtención del Índice de Incidencia a partir de los códigos asignados a los atributos

Magnitud del impacto. La valoración cuantitativa de la magnitud se realiza por medio de indicadores de impacto que permiten medir cuantitativamente los efectos de una acción sobre un factor ambiental en unidades heterogéneas (incomparables, por tanto), y de funciones de transformación que permiten transformar las medidas anteriores en valores adimensionales (comparables, por tanto), de impacto ambiental, los cuales varían entre un mínimo "0" y un máximo "1".

El programa contiene por defecto, más de 300 indicadores de impacto y para cada uno de ellos su correspondiente función de transformación; el usuario podrá utilizarlos directamente, modificarlos o crear sus propios indicadores y funciones para los impactos que considere conveniente. Las tareas por realizar en esta fase, se limitan a elegir el indicador más adecuado (o definir uno nuevo), y estimar los valores que toma este indicador en la situación "sin" y "con" proyecto. El resultado se denomina Valor de la Magnitud del Impacto. 
Impacto $\mathrm{N}^{\circ} 15$, Disminución del confort sonoro.

Seleccione el indicador que desea utilizar.

\begin{tabular}{lll|}
\hline Indicadores Generales & - Indicadores Propios \\
1-Aire-Seleccionar indicador &
\end{tabular}

o crée un Indicador nuevo: Crear

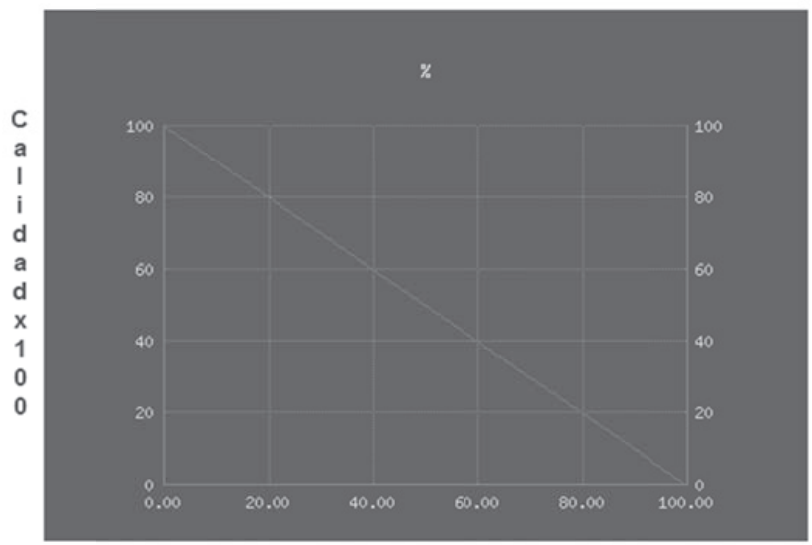

No 23. Porcentaje de personas afectadas por niveles
sonoros diurnos perjudiciales
Valor Indicador "sin" Proyecto: 0
Valor Indicador "con" Proyecto: 0
Valor calidad "sin" Proyecto:
Valor calidad "con" Proyecto:
Valor Magnitud:
Calcular

Figura 11. Representación gráfica de una función de transformación

En la Figura 11, se puede ver una función de transformación de un indicador tal como la muestra el programa, donde se señalan los datos que se deben introducir para la valoración cuantitativa de los impactos y los resultados obtenidos.

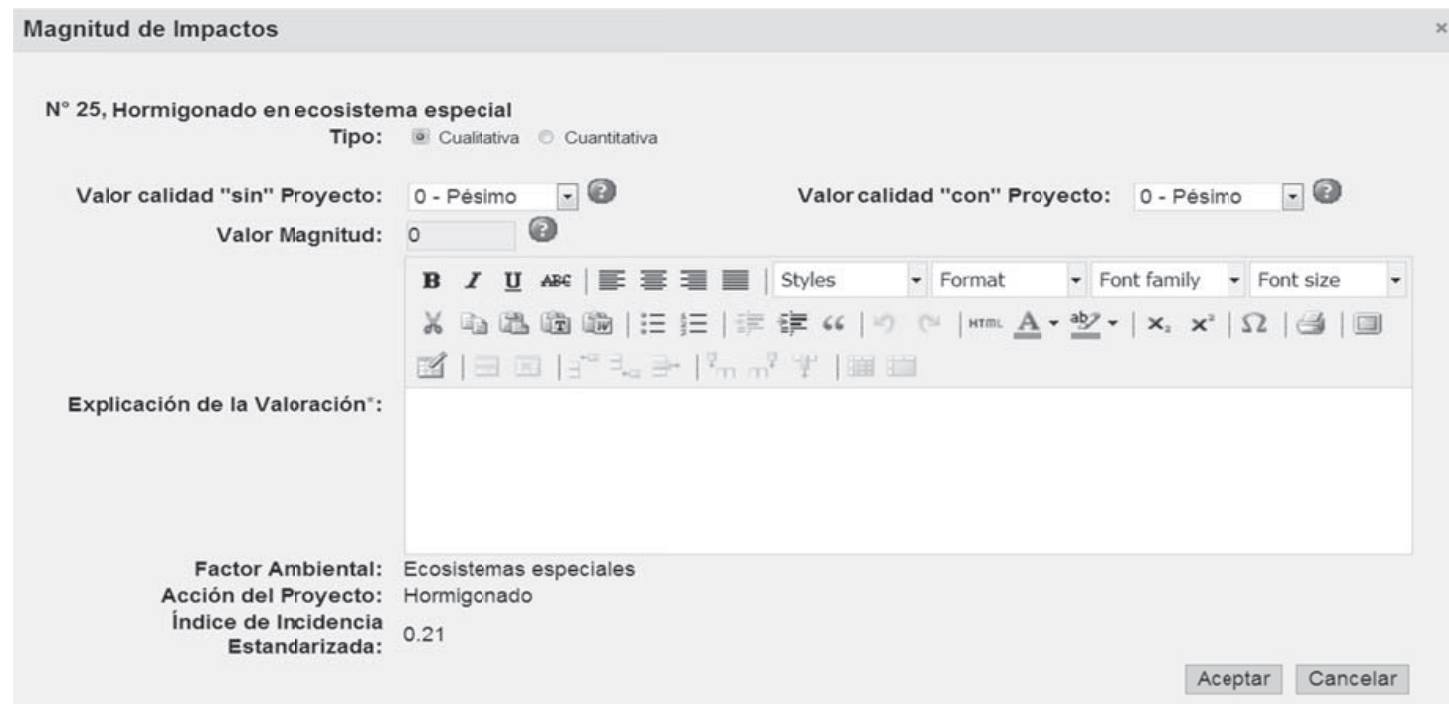

Figura 12. Valoración cualitativa de la magnitud de los impactos 
En cambio, la valoración cualitativa (Figura 12), permite valorar con más rapidez los impactos, pero de forma menos rigurosa y objetiva que en la cuantitativa; el usuario decidirá el método que utiliza en cada caso; la valoración puede ser directa o por medio de dos índices: el de incidencia y el de magnitud, ambos valorados cualitativamente.

\subsection{Enjuiciamiento de los impactos y totalización final del impacto del proyecto}

La emisión del juicio de cada impacto, se realiza en función de la información que presenta la pantalla, siendo las categorías de enjuiciamiento, las definidas por la legislación: Positivo, Compatible, Moderado, Severo y Crítico (Figura 13). Aparecerá en la pantalla, la Tabla de Enjuiciamiento con los impactos identificados según la leyenda:

- Gris: Sin enjuiciar

- Verde: Positivo Significativo

- Amarillo: Compatible $(I \times M<0,15)$

- Naranja: Moderado(0,15 $\leq$ I x $M<0,36)$

- Rojo: Severo $(0,36 \leq 1 \times M<0,65)$

- Morado: Crítico $(0,65 \leq 1 \times M)$

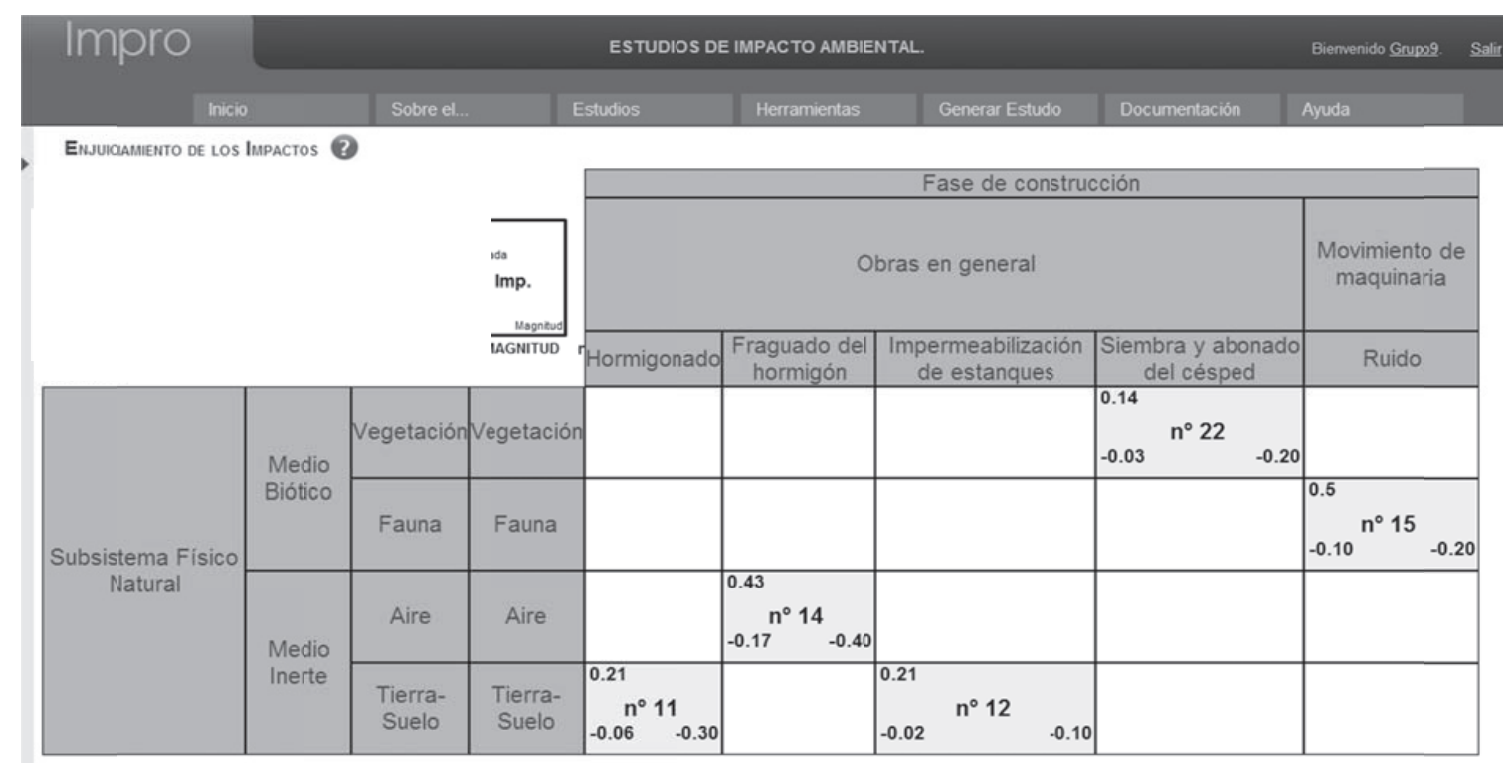

Figura 13. Ventana de enjuiciamiento de los impactos

Por último, se obtiene el Impacto Total del Proyecto por suma ponderada de todos los impactos sobre los diferentes factores afectados, multiplicados por el peso que se les haya atribuido. Como los pesos de los factores se ajustan a suma 1.000 y cada impacto varía entre 0 y 1 , el impacto total de un proyecto estará siempre comprendido entre 0 y 1.000 unidades de impacto. 


\subsection{Prevención de Impactos}

Para definir las medidas para la prevención, mitigación, corrección y/o compensación del impacto, el programa plantea un sencillo cuestionario donde debe identificarse el nombre de la medida, la acción causante del impacto, el factor afectado, la descripción del efecto, el carácter y el objetivo que se pretende con la medida.

\subsection{Programa de Vigilancia Ambiental}

El programa permite elaborar la parte del Programa de Vigilancia Ambiental referente al seguimiento de las medidas de mitigación.

\subsection{Generación del Estudio}

Impro4-ElA permite exportar al formato “.doc" de Microsoft Word, todo el contenido visualizable, de forma que cualquier usuario con un editor de texto compatible, pueda realizar los ajustes y cambios que considere necesarios antes de su impresión definitiva.

\section{CONCLUSIONES}

Como se ha visto, la aplicación informática Impro4-EIA permite:

- Definir detalladamente el proyecto y trabajar con varias alternativas.

- Identificar de manera sistemática los posibles efectos del proyecto, caracterizándolos en los términos que establece la legislación de EIA.

- Valorar cuantitativa y cualitativamente los impactos.

- Establecer medidas correctoras de los impactos y el Programa de Vigilancia Ambiental.

- Con base en todos los datos, emitir un juicio en los términos que establece la legislación vigente.

- Exportar el documento con los resultados del Estudio a formatos como doc, xls u otros, para imprimirlo y presentarlo ante la Autoridad Ambiental.

Es decir, Impro4-ElA automatiza las tareas que aportan poco o ningún valor añadido, permitiendo al profesional dedicar todo su tiempo a las actividades más sustanciosas. Así mismo, permite:

- Mejorar el contenido, metodología y calidad de los trabajos.

- Asegurar que la documentación que se elabore cumpla los requisitos legales.

- Optimizar el acceso, gestión y tratamiento de la información entre los agentes que intervienen en la EIA.

- Posibilidad de compartir y colaborar con profesionales de distintos países.

- Posibilidad de divulgar los trabajos realizados. 
De este modo, la aplicación Impro4 se convierte en una herramienta útil, tanto en la práctica profesional, como en la investigación y la docencia.

\section{REFERENCIAS BIBLIOGRÁFICAS}

[1] Gómez Orea D., (2003). Evaluación de Impacto Ambiental. Ed. Mundi Prensa. Madrid.

[2] Canter L., (1998). Manual de evaluación de impacto ambiental. Técnicas para la elaboración de Estudios de Impacto. McGraw-Hill. Madrid.

[3] García Álvarez A., (1994). Guía práctica de evaluación de impacto ambiental. Ed. Amaru. Madrid.

[4] Conesa Fernández V., (2000). Guía metodológica de evaluación de impacto ambiental. Ed. Mundi Prensa. Madrid.

[5] Dirección General de Medio Ambiente. Ministerio de Obras Públicas y Urbanismo (1989). Guías metodológicas para la elaboración de estudios de impacto ambiental. Diversos casos. Madrid.

[6] Instituto Tecnológico Geominero de España. Ministerio de Industria y Energía; (1992). Evaluación y corrección de impactos ambientales. Instituto Tecnológico Geominero de España. Madrid.

[7] Leopold L.B. et al., (1973). A procedure for Evaluating Environmental Impact. US Departament of the Interior. Gov. Print. Office.

[8] Hernández Fernández S., (1999). La legislación de evaluación de impacto ambiental en España. Ed. Mundi Prensa. Madrid.

[9] Gómez Orea D., (2007). Evaluación ambiental estratégica: un instrumento para integrar el medio ambiente en la elaboración de planes y programas. Ed. Mundi Prensa. Madrid. 
